\title{
Commentary
}

\section{Influenza Virus Receptor Specificity}

\section{Disease and Transmission}

\author{
Adolfo García-Sastre \\ From the Department of Microbiology and the Global Health and \\ Emerging Pathogens Institute, Mount Sinai School of Medicine, \\ New York, New York
}

To be successful, respiratory viruses must efficiently infect their hosts through the respiratory mucosa, replicate, and be shed in the appropriate areas of the respiratory tract in the form of highly infectious transmissible material that infects a new host. The interaction between the viral attachment protein and its cellular receptor are among the critical molecular determinants that regulate respiratory virus infection, replication, and shedding, and therefore transmission. Thus, the presence or absence of cellular receptors in specific hosts and tissues is one of the factors that determines whether the host is susceptible or not to viral infection, the tissues and cell types where the virus replicates, and the route of viral transmission.

For influenza viruses, it has been known for a long time that the viral attachment protein, hemagglutinin (HA), binds to and uses sialic acid-containing molecules as receptors. The use of such abundant and ubiquitous molecules as receptors, while providing the apparent advantage to the virus of allowing infection of multiple cell types and species, also results in binding to nonproductive receptors present in respiratory secretions, surfaces of dead cells, and even other virions. It is because of this capability that influenza virus has evolved a second viral surface protein, neuraminidase, as a receptor-destroying enzyme that cleaves sialic acid, allowing the virus to be released after binding to sialic acid-containing molecules that do not lead to viral infection.

This picture of multiple interactions of the influenza virus with its receptor is further complicated by early findings indicating that not all sialic acid-containing molecules bind with equal efficiency to HA and that different viral strains show different receptor specificities according to their host tropism. Most influenza viruses circulate in waterfowl, and these avian influenza virus strains contain an HA with preference for binding to sialic acids linked to the rest of the sugar by an $\alpha 2-3$ linkage. In contrast, HAs from human influenza virus strains show enhanced binding to $\alpha 2-6$-linked sialic acids. ${ }^{1}$ This correlates with an abundance of $\alpha 2-6$-linked sialic acids in the upper respiratory tract of humans, and of $\alpha 2-3-$ linked sialic acids in the intestinal mucosa of birds, where replication of human and avian strains of influenza viruses takes place, respectively. ${ }^{2}$ More detailed analysis of the abundance of these types of linkages in the whole human respiratory tract and in the context of severe infection in humans with highly pathogenic avian H5N1 influenza viruses led to the conclusion that $\alpha 2-3$-linked sialic acids are more abundant in the human lower respiratory tract, which correlates with an enhanced tropism of H5N1 viruses for deep areas in the human lung. This may contribute to both severe disease, as viral replication in the lower respiratory tract is more likely to induce pneumonia, as well as to the lack of efficient transmission of H5N1 viruses from human to human, because the virus is less likely to infect the upper respiratory tract, where $\alpha 2-3-$ linked sialic acids are sparse. ${ }^{3,4}$ Consistent with a role of sialic acid binding preference in transmission, it was found that changes in receptor specificity in the $\mathrm{HA}$ of the 1918 human $\mathrm{H} 1 \mathrm{~N} 1$ pandemic influenza virus from $\alpha 2-6$ to $\alpha 2-3$ linkages dramatically decreased its aerosol transmission in the ferret model, although surprisingly this was not accompanied with decreased viral shedding. ${ }^{5}$ Thus, whether more efficient HA binding to $\alpha 2-6$-linked sialic acids results in an increased tropism for the human upper respiratory tract and in increased viral transmission in humans is an attractive hypothesis that still requires more research to be proven.

In this issue of The American Journal of Pathology, van Riel et $\mathrm{al}^{6}$ have provided new evidence that supports this hypothesis. Using three representative human influenza A virus strains, corresponding to seasonal H1N1, H3N2, and the new pandemic $\mathrm{H} 1 \mathrm{~N} 1$ viruses, and three avian influenza viruses of the $\mathrm{H} 5$ and $\mathrm{H} 7$ subtypes, including a highly pathogenic H5N1 strain, the authors have analyzed the pattern of HA-mediated binding of virions to

Accepted for publication January 27, 2010.

Address reprint requests to Adolfo García-Sastre, Mount Sinai School of Medicine, Department of Microbiology, 1468 Madison Avenue, New York, NY 10029. E-mail: adolfo.garcia-sastre@mssm.edu. 
different human tissues of the upper respiratory tract. For this purpose, they have used a previously developed technique by the same group, named virus histochemistry. In this technique, influenza virions are labeled with FITC, and on incubation with fixed human tissue sections, virions that remain bound are visualized using a peroxidase-labeled anti-FITC secondary antibody. ${ }^{4}$ All human influenza virus strains readily attached and decorated ciliated epithelial and goblet cells from human upper respiratory tract tissues, such as nasal septum, concha, and nasopharynx. In contrast, avian influenza viruses were poor binders to the same tissues. Interestingly, the same group has previously shown that avian influenza viruses are nevertheless able to bind well to cells in tissue sections from the human lower respiratory tract, especially to type II pneumocytes and to alveolar macrophages, whereas human influenza viruses have preference for type I pneumocytes and rarely bind to type II pneumocytes and macrophages. ${ }^{7}$ Taking both observations together, a pattern emerges where binding to macrophages and type II pneumocytes in the lower respiratory tract may promote infection of these cells and destruction of alveolar structures and induction of high levels of cytokines, leading to severe disease, but at the expense of loosing binding to and viral replication in cells of the upper respiratory tract, which is likely to be essential for transmission. If this is the case, the most pathogenic influenza viruses for humans are also the less transmissible.

An important advance of the study by van Riel et al ${ }^{6}$ is the comprehensive analysis that has been conducted using multiple human tissue samples derived from the respiratory tract. This provides a fingerprint pattern for influenza viruses that efficiently transmit in humans or that are more likely to induce lower respiratory disease. Although it is clear that host tropism and virulence is dependent of multiple virus and host factors, and not only of HA receptor specificity, virus histochemisty might give the first indication whether a particular viral strain is more likely to transmit in humans or to cause severe disease in humans. For example, some severe cases of influenza virus infection with the new pandemic $\mathrm{H} 1 \mathrm{~N} 1$ virus have been correlated with the presence of specific mutations in the HA, and it will be interesting to use virus histochemistry to compare the pattern of binding of these mutant viruses to human respiratory tissue. Importantly, as the hallmark of pandemic influenza consists in the introduction in humans of a novel virus strain expressing an HA derived from an animal strain for which there is little pre-existing immunity in humans, efficient transmission in humans of the new pandemic virus requires that its HA binds to sialic acid-containing receptors present in cells of the human upper respiratory tract.

However, there are still many unknowns with respect to the relationship between HA receptor specificity and influenza virus host and tissue tropism. It is, for example, quite clear now that the determinants of influenza tropism are more complex than the simplistic early view of classifying HAs by preferential binding to $\alpha 2-6$ and $\alpha 2-3$ sialic acids. The development of glycan arrays that allow to determine more precisely the ability of influenza viruses to bind to different sugar molecules have demonstrated a wide variety of complex binding patterns according to the specific viral strain. ${ }^{8}$ It is now clear that not only the linkage between the sialic acid and the next sugar influences binding of a specific viral and/or HA strain, but also the type of sialic acid as well as the rest of the carbohydrate. Because both the distribution of all possible different sialic acid-containing sugars in respiratory tissues and the types of molecules that can be used as receptors for productive infection by influenza viruses remain unclear, it remains to be determined what the different patterns of binding to specific carbohydrates by different influenza virus strains mean. It is also not known whether high affinity binding necessarily correlates with high infectivity, as this might inhibit viral spreading by facilitating virus retention in noninfectable surfaces of the respiratory tract. Finally, whether infection of specific cells in the respiratory tract facilitates virus mobilization into infectious aerosols and respiratory droplets, and therefore also facilitates transmission, is again unclear. Thus, more research is required to understand how the complexity of interactions of influenza viruses with their receptor determines the outcome of viral infection and transmission. A better understanding of these processes might facilitate the design of specific antivirals that stop influenza virus transmission and infection of the lower respiratory tract.

\section{References}

1. Connor RJ, Kawaoka Y, Webster RG, Paulson JC: Receptor specificity in human, avian, and equine $\mathrm{H}_{2}$ and $\mathrm{H} 3$ influenza virus isolates. Virology 1994, 205:17-23

2. Baum LG, Paulson JC: Sialyloligosaccharides of the respiratory epithelium in the selection of human influenza virus receptor specificity. Acta Histochem Suppl 1990, 40:35-38

3. Shinya K, Ebina M, Yamada S, Ono M, Kasai N, Kawaoka Y: Avian flu: influenza virus receptors in the human airway. Nature 2006, 440: 435-436

4. van Riel D, Munster VJ, de Wit E, Rimmelzwaan GF, Fouchier RA, Osterhaus AD, Kuiken T: H5N1 Virus Attachment to Lower Respiratory Tract. Science 2006, 312:399

5. Tumpey TM, Maines TR, Van Hoeven N, Glaser L, Solorzano A, Pappas C, Cox NJ, Swayne DE, Palese P, Katz JM, García-Sastre A: A twoamino acid change in the hemagglutinin of the 1918 influenza virus abolishes transmission. Science 2007, 315:655-659

6. van Riel D, den Bakker M, Leijten L, Chutinimitkul S, Munster VJ, de Wit E, Rimmelzwaan GF, Fouchier RAM, Osterhaus ADME, Kuiken T: Seasonal and pandemic human influenza virus attach better to human upper respiratory tract epithelium than avian influenza viruses. Am J Pathol 2010, 176:1614-1618

7. van Riel D, Munster VJ, de Wit E, Rimmelzwaan GF, Fouchier RA Osterhaus $A D$, Kuiken $T$ : Human and avian influenza viruses target different cells in the lower respiratory tract of humans and other mammals. Am J Pathol 2007, 171:1215-1223

8. Stevens J, Blixt O, Paulson JC, Wilson IA: Glycan microarray technologies: tools to survey host specificity of influenza viruses. Nat Rev Microbiol 2006, 4:857-864 